\title{
Stochastic Localization + Stieltjes Barrier $=$ Tight Bound for Log-Sobolev
}

\author{
Yin Tat Lee \\ University of Washington and Microsoft Research \\ Seattle, WA, USA \\ yintat@uw.edu
}

\author{
Santosh S. Vempala \\ Georgia Tech \\ Atlanta, GA, USA \\ vempala@gatech.edu
}

\begin{abstract}
Logarithmic Sobolev inequalities are a powerful way to estimate the rate of convergence of Markov chains and to derive concentration inequalities on distributions. We prove that the log-Sobolev constant of any isotropic logconcave density in $\mathbb{R}^{n}$ with support of diameter $D$ is $\Omega(1 / D)$, resolving a question posed by Frieze and Kannan in 1997 . This is asymptotically the best possible estimate and improves on the previous bound of $\Omega\left(1 / D^{2}\right)$ by Kannan-LovászMontenegro. It follows that for any isotropic logconcave density, the ball walk with step size $\delta=\Theta(1 / \sqrt{n})$ mixes in $O\left(n^{2} D\right)$ proper steps from any starting point. This improves on the previous best bound of $O\left(n^{2} D^{2}\right)$ and is also asymptotically tight. The new bound leads to the following refined large deviation inequality for a $L$ Lipschitz function $g$ over an isotropic logconcave density $p$ : for any $t>0$,

$$
\mathbb{P}_{x \sim p}(|g(x)-\bar{g}| \geq c \cdot L \cdot t) \leq \exp \left(-\frac{t^{2}}{t+\sqrt{n}}\right)
$$

where $\bar{g}$ is the median or mean of $g$ for $x \sim p$; this improves on previous bounds by Paouris and by Guedon-Milman. Our main proof is based on stochastic localization together with a Stieltjestype barrier function ${ }^{1}$.
\end{abstract}

\section{CCS CONCEPTS}

- Mathematics of computing $\rightarrow$ Multivariate statistics; Functional analysis; Mathematical analysis; • Theory of computation $\rightarrow$ Random walks and Markov chains;

\section{KEYWORDS}

Log-Sobolev constant, Logconcave densities, stochastic localization, Stieltjes barrier, ball walk

\section{ACM Reference Format:}

Yin Tat Lee and Santosh S. Vempala. 2018. Stochastic Localization + Stieltjes Barrier = Tight Bound for Log-Sobolev. In Proceedings of 50th Annual ACM SIGACT Symposium on the Theory of Computing (STOC'18). ACM, New York, NY, USA, 8 pages. https://doi.org/10.1145/3188745.3188866

${ }^{1}$ Full version of paper with all proofs is on the arXiv at https://arxiv.org/abs/1712.01791.

Permission to make digital or hard copies of all or part of this work for personal or classroom use is granted without fee provided that copies are not made or distributed for profit or commercial advantage and that copies bear this notice and the full citation on the first page. Copyrights for components of this work owned by others than ACM must be honored. Abstracting with credit is permitted. To copy otherwise, or republish to post on servers or to redistribute to lists, requires prior specific permission and/or a fee. Request permissions from permissions@acm.org.

STOC'18, June 25-29, 2018, Los Angeles, CA, USA

(C) 2018 Association for Computing Machinery.

ACM ISBN 978-1-4503-5559-9/18/06 . \$ \$15.00

https://doi.org/10.1145/3188745.3188866

\section{INTRODUCTION}

This purpose of this paper is to understand the asymptotic behavior of the log-Sobolev and log-Cheeger constants of convex bodies and logconcave distributions in $\mathbb{R}^{n}$. These fundamental parameters, which we will define presently, have many important connections and applications (cf. [15]). To introduce them, we first remind the reader of the Cheeger constant (a.k.a. isoperimetric constant or expansion).

Definition 1.1. For a density $p$ in $\mathbb{R}^{n}$, the Cheeger constant of $p$ is defined as

$$
\psi_{p} \stackrel{\text { def }}{=} \inf _{S \subseteq \mathbb{R}^{n}} \frac{\int_{\partial S} p(x) d x}{\min \left\{\int_{S} p(s) d x, \int_{\mathbb{R}^{n} \backslash S} p(x) d x\right\}} .
$$

Kannan, Lovász and Simonovits [12] conjectured that for any logconcave density, the Cheeger constant satisfies ${ }^{2} \psi_{p} \gtrsim\|A\|_{\mathrm{op}}^{-1 / 2}$ where $A$ is the covariance matrix of $p$. A density or distribution is called isotropic if its covariance matrix is the identity, a normalization that can be achieved via an affine transformation. For isotropic logconcave densities, the conjecture says the Cheeger constant is $\Omega(1)$. The current best estimate of $\psi_{p}$ is the following recent result.

Theorem 1.2 ([18]). For any logconcave density $p$ in $\mathbb{R}^{n}$ with covariance matrix $A$,

$$
\psi_{p} \gtrsim\left(\operatorname{Tr}\left(A^{2}\right)\right)^{-1 / 4}
$$

For isotropic $p$, this gives a bound of $\psi_{p} \gtrsim n^{-\frac{1}{4}}$. The KLS hyperplane conjecture plays a central role in asymptotic convex geometry, and implies several other well-known (older) conjectures, including slicing (or hyperplane) and thin-shell (or variance) conjectures, the Poincare conjecture, central limit, exponential concentration etc. (see e.g., [4]).

The KLS conjecture was motivated by the study of the convergence of a Markov chain, the ball walk in a convex body. To sample uniformly from a convex body, the ball walk starts at some point in the body, picks a random point in the ball of radius $\delta$ around the current point and if the chosen point is in the body, it steps to the new point. It can be generalized to sampling any logconcave density by using a Metropolis filter. As shown in [13], the ball walk applied to a logconcave density mixes in $O^{*}\left(n^{2} / \psi_{p}^{2}\right)$ steps from a warm start, which using the current-best bound [18] is $O^{*}\left(n^{2.5}\right)$. Looking closer, from a starting distribution $Q_{o}$, the distance of the distribution obtained after $t$ steps from $Q_{o}$ to the stationary

${ }^{2}$ We write $\gtrsim$ to denote "at least a constant times". 
distribution $Q$ drops as

$$
d\left(Q_{t}, Q\right) \leq d\left(Q_{0}, Q\right)\left(1-\frac{\phi^{2}}{2}\right)^{t}
$$

where $\phi$ is the conductance of the Markov chain and $d(.,$.$) is the$ $\chi$-squared distance. The conductance can be viewed as the Cheeger constant of the Markov chain. Thus the number of steps needed is $O\left(\phi^{-2} \log \left(1 / d\left(Q_{0}, Q\right)\right)\right)$. Roughly speaking, for the ball walk applied to a logconcave density $p$, the conductance is $\Omega\left(\psi_{p} / n\right)$, leading to the bound of $O^{*}\left(n^{2} / \psi_{p}^{2}\right)$ steps from a warm start. The dependence on the starting distribution leads to an additional factor of $n$ in the running time when the starting distribution is not warm (i.e., $d\left(Q_{1}, Q\right)$ after one step can be $\left.e^{\tilde{\Omega}(n)}\right)$. This is a general issue for Markov chains. One way to address this is via the log-Sobolev constant $[6,15]$. We first define it for a density, then for a Markov chain.

Definition 1.3. For a density $p$ the log-Sobolev constant $\rho_{p}$ is the largest $\rho$ such that for every smooth function $f: \mathbb{R}^{n} \rightarrow \mathbb{R}$ with $\int f^{2} d p=1$, we have

$$
\rho \leq \frac{2 \int\|\nabla f\|^{2} d p}{\int f^{2} \log f^{2} d p} .
$$

A closely related parameter is the following.

Definition 1.4. The log-Cheeger or log-isoperimetric constant $\kappa_{p}$ of a density $p$ in $\mathbb{R}^{n}$ is

$$
\kappa_{p}=\inf _{S \subseteq \mathbb{R}^{n}} \frac{p(\partial S)}{\min \left\{p(S), p\left(\mathbb{R}^{n} \backslash S\right)\right\} \sqrt{\log \left(\frac{1}{p(S)}\right)}} .
$$

It is known that $\rho_{p}=\Theta\left(\kappa_{p}^{2}\right)$ (see e.g., [14]). The log-Cheeger constant shows more explicitly that the log-Sobolev constant is a uniform bound on the expansion "at every scale".

For a reversible Markov chain with transition operator $P$ and stationary density $Q$, the analogous definition is the infimum over all smooth functions satisfying $f: \mathbb{R}^{n} \rightarrow \mathbb{R}$ with $\int f^{2} d p=1$ of

$$
\rho(P)=\frac{\int_{x} \int_{y}\|f(x)-f(y)\|^{2} P(x, y) d Q(x)}{\int f^{2} \log f^{2} d Q} .
$$

Diaconis and Saloff-Coste [6] show that the distribution after $t$ steps satisfies $\operatorname{Ent}\left(Q_{t}\right) \leq e^{-4 \rho(P) t} \operatorname{Ent}\left(Q_{0}\right)$ where $\operatorname{Ent}\left(Q_{t}\right)=\int Q_{t} \log \frac{Q_{t}}{Q_{0}} d Q_{0}$ is the entropy with respect to the stationary distribution. Thus, the dependence of the mixing time on the starting distribution goes down from $\log \left(1 / d\left(Q_{0}, Q\right)\right)$ to $\left.\log \log \left(1 / d\left(Q_{0}, Q\right)\right)\right)$. Moreover, just as in the case of the Cheeger constant, for the ball walk, the Markov chain parameter is determined by the log-Sobelov constant $\rho_{p}$ for sampling from the density $p$. It is thus natural to ask for the best possible bound on $\rho_{p}$ or $\kappa_{p}$. Unlike the Cheeger constant, which is conjectured to be at least a constant for isotropic logconcave densities, it is known that $\rho_{p}$ cannot be bounded from below by a universal constant, in particular for distributions that are not " $\psi_{2}$ " (distributions with sub-Gaussian tail).

Kannan, Lovász and Montenegro [11] gave the following bound on $\kappa_{p}$. Our main result (Theorem 1.8) is an improvement of this bound to the best possible asymptotic bound.
Theorem 1.5 ([11]). For an isotropic logconcave density $K \subset \mathbb{R}^{n}$ with support of diameter $D$, we have $\kappa_{p} \gtrsim \frac{1}{D}$ and $\rho_{p} \gtrsim \frac{1}{D^{2}}$.

From the above bound, it follows that the ball walk mixes in $O\left(n^{2} D^{2}\right)$ proper steps of step size $\delta$. A proper step is one where the current point changes. For an isotropic logconcave density $\delta=\Theta\left(\frac{1}{\sqrt{n}}\right)$ is small enough so that the number of wasted steps is of the same order as the number of proper steps in expectation. Moreover, by restricting to a ball of radius $D=O(\sqrt{n})$, the resulting distribution remains near-isotropic and very close in total variation distance to the original. Together, this considerations imply a bound of $O^{*}\left(n^{3}\right)$ proper steps from any starting point as shown in [11]. Is this bound the best possible? From a warm start, the KLS conjecture implies a bound of $O^{*}\left(n^{2}\right)$ steps and current best bound is $O^{*}\left(n^{2.5}\right)$. Thus, the mixing of the ball walk, which was the primary motivation for formulation of the KLS conjecture, also provides a compelling reason to study the log-Sobolev constant. Estimating the log-Sobolev constant was posed as an open problem by Frieze and Kannan [8] when they analyzed the log-Sobolev constant of the grid walk to sample sufficiently smooth logconcave densities.

The Cheeger and log-Sobolev constants also play an important role in the phenomenon known as concentration of measure. The following result is due to Gromov and Milman.

Theorem 1.6 (Lipschitz Concentration [9]). For any L-Lipschitz function $g$ in $\mathbb{R}^{n}$, and isotropic logconcave density $p$,

$$
\mathbb{P}_{x \sim p}(|g(x)-\mathbb{E} g| \geq c \cdot L \cdot t) \leq e^{-t \psi_{p}} .
$$

Using the best-known estimate of the Cheeger constant gives a bound of $e^{-t / n^{1 / 4}}$ [18]. A different bound, independent of the Cheeger constant, for the deviation in length of a random vector was given in a celebrated paper by Paouris [20] and improved by Guedon and Milman [10] (Paouris' result has only the second term in the minimum below, and is sharp when $t \gtrsim \sqrt{n}$ ).

Theorem 1.7 ([10, 20]). For any isotropic logconcave density p,

$$
\mathbb{P}_{x \sim p}(|\|x\|-\sqrt{n}| \geq c \cdot t) \leq e^{-\min \left\{\frac{t^{3}}{n}, t\right\}} .
$$

Our tight log-Sobolev bound will be useful in proving an improved concentration inequality.

\subsection{Results}

Our main theorem is the following.

THEOREM 1.8. For any isotropic logconcave densityp with support of diameter $D$, the log-Cheeger constant satisfies $\kappa_{p} \gtrsim \frac{1}{\sqrt{D}}$ and the log-Sobolev constant satisfies $\rho_{p} \gtrsim \frac{1}{D}$.

As we show in Section 5, these bounds are asymptotically the best possible (Lemma 5.1). The improved bound has interesting consequences. The first is an improved concentration of mass inequality for logconcave densities. In particular, this gives an alternative proof of Paouris' (optimal) inequality [20] for the large deviation case $(t \geq \sqrt{n})$.

THeorem 1.9. For any L-Lipschitz function $g$ in $\mathbb{R}^{n}$ and any isotropic logconcave density $p$, we have that

$$
\mathbb{P}_{x \sim p}(|g(x)-\bar{g}(x)| \geq c \cdot L \cdot t) \leq \exp \left(-\frac{t^{2}}{t+\sqrt{n}}\right)
$$


where $\bar{g}$ is the median or mean of $g(x)$ for $x \sim p$.

As mentioned earlier, the previous best bound is

$$
\exp \left(-\min \left(\frac{t^{3}}{n}, t\right)\right)
$$

for the function $g(x)=\|x\|[10]$ and $\exp \left(-\frac{t}{n^{1 / 4}}\right)$ for a general Lipschitz function $g$ [18]. The new bound can be viewed as an improvement and generalization of both (note that we can write the exponent as $\left.-\min \left(\frac{t^{2}}{n}, t\right)\right)$. Also this concentration result does not need bounded support for the density $p$.

For the next consequence, we circle back to the analysis of the ball walk to resolve the open problem posed by Frieze and Kannan [8].

THEOREM 1.10. The ball walk with step size $\delta=\Theta(1 / \sqrt{n})$ applied to an isotropic logconcave density in $\mathbb{R}^{n}$ with support of diameter $D$ mixes in $\mathrm{O}^{*}\left(n^{2} D\right)$ proper steps from any starting point of positive density (or from any interior point of a convex body).

The choice of $\delta=\Theta(1 / \sqrt{n})$ is optimal up to a constant factor for isotropic logconcave distributions (Lemma 5.2). The bound on the number of steps improves on the previous best bound of $O^{*}\left(n^{2} D^{2}\right)$ proper steps for the mixing of the ball walk from an arbitrary starting point [11] and as we show in Section 5, $O\left(n^{2} D\right)$ is the best possible bound. For sampling, we can restrict the density to a ball of radius $O(\sqrt{n})$ losing only a negligibly small measure, so the bound is $O\left(n^{2.5}\right)$ from an arbitrary starting point, which matches the current best bound from a warm start. The mixing time from a warm start for an isotropic logconcave density is $O\left(n^{2} \psi_{p}^{2}\right)$, or $O\left(n^{2}\right)$ if the KLS conjecture is true; but from an arbitrary start, our analysis is essentially the best possible, independent of any further progress on the conjecture!

\section{APPROACH: STOCHASTIC LOCALIZATION}

In this section, we describe the stochastic localization method introduced by Eldan [7], and, in particular, the variant of the method used in [18]. The idea of the approach is to gradually modify the density $p$ by making infinitesimal changes so that the measure of a set and its boundary is not changed by much, but the density function itself accumulates a significant Gaussian component, i.e., it looks like a Gaussian density function times a logconcave function. For such densities, we can use standard localization (or other methods) to prove that the log-Sobolev constant is large. While this is the same high-level approach as in previous papers, several new challenges arise. First, unlike previous applications, we cannot simply work with subsets of measure $1 / 2$ or a constant, it is crucial to consider arbitrarily small subsets. Second, as we will discuss presently, we need a more refined potential function to understand the evolution of the measure.

Definition 2.1. Given a logconcave distribution $p$, we define the following stochastic differential equation:

$$
c_{0}=0, \quad d c_{t}=d W_{t}+\mu_{t} d t,
$$

where $W_{t}$ is the Wiener process and the probability distribution $p_{t}$ is defined by

$$
p_{t}(x)=\frac{e^{c_{t}^{T} x-\frac{t}{2}\|x\|_{2}^{2}} p(x)}{\int_{\mathbb{R}^{n}} e^{c_{t}^{T} y-\frac{t}{2}\|y\|_{2}^{2}} p(y) d y} .
$$

The mean $\mu_{t}$ and the covariance $A_{t}$ are defined by

$$
\mu_{t}=\mathbb{E}_{x \sim p_{t}} x, \quad A_{t}=\mathbb{E}_{x \sim p_{t}}\left(x-\mu_{t}\right)\left(x-\mu_{t}\right)^{T} .
$$

We collect the properties of this stochastic localization that we will use in this paper in the following Lemma.

Lemma 2.2 ([18]). For any logconcave distribution $p$ with bounded support, the stochastic process $p_{t}$ defined in Definition 2.1 exists and is unique. Also, $p_{t}$ is a martingale, and in particular, for any $x \in \mathbb{R}^{n}$

$$
d p_{t}(x)=\left(x-\mu_{t}\right)^{T} d W_{t} \cdot p_{t}(x) .
$$

Its covariance matrix satisfies

$$
d A_{t}=\int_{\mathbb{R}^{n}}\left(x-\mu_{t}\right)\left(x-\mu_{t}\right)^{T}\left(\left(x-\mu_{t}\right)^{T} d W_{t}\right) \cdot p_{t}(x) d x-A_{t}^{2} d t .
$$

In previous papers $[7,18]$, the spectral norm of the covariance the $\left\|A_{t}\right\|_{\text {op }}$ is bounded via a potential function of the form $\operatorname{Tr}\left(A_{t}^{q}\right)$. However, to obtain a tight result without extraneous logarithmic factors, we study the Stieltjes-type potential $\operatorname{Tr}\left(\left(u I-A_{t}\right)^{-q}\right)$.

To define the potential, fix integers $n, q \geq 1$ and a positive number $\Phi>0$. Let $u(X)$ be the real-valued function on $n \times n$ symmetric matrices defined by the solution of the following equation

$$
\operatorname{Tr}\left((u I-X)^{-q}\right)=\Phi \text { and } X \leq u I
$$

Note that this is the same as the solution to $\sum_{i=1}^{n} \frac{1}{\left(u-\lambda_{i}\right)^{q}}=\Phi$ and $\lambda_{i} \leq u$ for all $i$ where $\lambda_{i}$ are the eigenvalues of $X$. Similar potentials have been used to analyze empirical covariance estimation [21], to build graph sparsifiers $[1,3,16,17]$ and to solve bandit problems [2].

The proof has the following ingredients:

(1) We show that for time $t$ up to $O\left(n^{-\frac{1}{2}}\right)$, the spectral norm of the covariance stays bounded (by a constant, say 2) with large probability (Lemma 3.2). This requires the use of the Stieltjes-type potential function.

(2) Then we consider any measurable subset $S$, with $g_{0}=p_{0}(S)$ and analyze its measure at time $t$, i.e., $g_{t}=p_{t}(S)$. In particular we show that up to time $\left(\log g_{0}+D\right)^{-1}$, the expectation of $g_{t} \sqrt{\log \left(1 / g_{t}\right)}$ remains large, i.e., a constant factor times its initial value (Lemma 3.8).

(3) The density at time $t$ has a Gaussian component of variance $1 / t$. For such a distribution, the log-Cheeger constant is $\Omega(\sqrt{t})$ (Theorem 3.9).

Together these facts will imply the main theorem.

\section{MAIN PROOF}

The goal of this section is to prove the following theorem, which implies Theorem 1.8. 
THeOREM 3.1. Given an isotropic logconcave distribution $p$ with support of diameter $D$. Then, for any measurable subset $S$,

$$
p(\partial S) \geq \Omega\left(\frac{\log \frac{1}{p(S)}}{D}+\sqrt{\frac{\log \frac{1}{p(S)}}{D}}\right) p(S) .
$$

\subsection{Bounding the Spectral Norm of the Covariance Matrix}

The main lemma of this section is the following.

LEMmA 3.2. There is some universal constant $c \geq 0$ such that for any $0 \leq T \leq \frac{1}{c \cdot \sqrt{n}}$, we have that

$$
\mathbb{P}\left(\max _{t \in[0, T]}\left\|A_{t}\right\|_{2} \geq 2\right) \leq 2 \exp \left(-\frac{1}{c T}\right) .
$$

We defer the proof of the following lemma to the end of this section.

LEMMA 3.3. We have that

$$
d u\left(A_{t}\right)=\alpha_{t}^{T} d W_{t}+\beta_{t} d t
$$

where

$$
\begin{aligned}
& \alpha_{t}=\frac{1}{\kappa_{t}} \mathbb{E}_{x \sim \tilde{p}_{t}} x^{T}\left(u I-A_{t}\right)^{-(q+1)} x \cdot x, \\
& \frac{\beta_{t}}{q+1} \leq \\
& \frac{1}{2 \kappa_{t}} \mathbb{E}_{x, y \sim \tilde{p}_{t}} x^{T}\left(u I-A_{t}\right)^{-1} y \cdot x^{T}\left(u I-A_{t}\right)^{-(q+1)} y \cdot x^{T} y \\
& -\frac{1}{\kappa_{t}^{2}} \mathbb{E}_{x, y \sim \tilde{p}_{t}} x^{T}\left(u I-A_{t}\right)^{-(q+1)} x \cdot y^{T}\left(u I-A_{t}\right)^{-(q+2)} y \cdot x^{T} y \\
& +\frac{1}{2 \kappa_{t}^{3}} \operatorname{Tr}\left(\left(u I-A_{t}\right)^{-(q+2)}\right) \cdot \mathbb{E}_{x, y \sim \tilde{p}_{t}} x^{T}\left(u I-A_{t}\right)^{-(q+1)} x . \\
& \cdot y^{T}\left(u I-A_{t}\right)^{-(q+1)} y \cdot x^{T} y, \\
& \kappa_{t}=\operatorname{Tr}\left(\left(u I-A_{t}\right)^{-(q+1)}\right) \\
& \text { and } \tilde{p}_{t} \text { be the translation of } p_{t} \text { defined by } \tilde{p}_{t}(x)=p_{t}\left(x+\mu_{t}\right) .
\end{aligned}
$$

To estimate $\alpha_{t}$, we need the following lemma proved in [18].

LeMma 3.4 ([18, Lemma 25]). Given a logconcave distribution $p$ with mean $\mu$ and covariance $A$. For any $C \geq 0$, we have that

$$
\left\|\mathbb{E}_{x \sim p}(x-\mu)^{T} C(x-\mu)(x-\mu)\right\|_{2}=O\left(\|A\|_{\text {op }}^{1 / 2}\right) \operatorname{Tr}\left(A^{1 / 2} C A^{1 / 2}\right) .
$$

To estimate $\beta_{t}$, we prove the following bound that crucially uses the KLS bound for non-isotropic logconcave distribution (Theorem 1.2).

Lemma 3.5. Given a logconcave distribution $p$ with mean $\mu$ and covariance $A$. For any $B^{(1)}, B^{(2)}, B^{(3)} \geq 0$,

$$
\begin{aligned}
& \left|\mathbb{E}_{x, y \sim p}(x-\mu)^{T} B^{(1)}(y-\mu) \cdot(x-\mu)^{T} B^{(2)}(y-\mu) \cdot(x-\mu)^{T} B^{(3)}(y-\mu)\right| \\
& \leq O(1) \cdot \operatorname{Tr}\left(A^{\frac{1}{2}} B^{(1)} A^{\frac{1}{2}}\right) \cdot\left\|A^{\frac{1}{2}} B^{(2)} A^{\frac{1}{2}}\right\|_{F} \cdot\left\|A^{\frac{1}{2}} B^{(3)} A^{\frac{1}{2}}\right\|_{\text {op }} .
\end{aligned}
$$$$
\text { For } q=2 \text {, let } u_{t}=u\left(A_{t}\right) \text {, we have that }
$$

$$
d u_{t}=\alpha_{t}^{T} d W_{t}+\beta_{t} d t
$$

with

$$
\left\|\alpha_{t}\right\|_{2} \leq O\left(u_{t}^{\frac{3}{2}}\right) \quad \text { and } \quad \beta_{t} \leq O\left(u_{t}^{3}\right) \sqrt{\Phi} .
$$

Now, we are ready to upper bound $\left\|A_{t}\right\|_{\mathrm{op}}$.

Proof of Lemma 3.2. Consider the potential $\Psi_{t}=-\left(u_{t}+1\right)^{-2}$. Using Lemma 3.5, we have that

$$
\begin{aligned}
d \Psi_{t} & =2\left(u_{t}+1\right)^{-3}\left(\alpha_{t}^{T} d W_{t}+\beta_{t} d t\right)-3\left(u_{t}+1\right)^{-4}\left\|\alpha_{t}\right\|^{2} d t \\
& \stackrel{\text { def }}{=} \gamma_{t}^{T} d W_{t}+\eta_{t} d t .
\end{aligned}
$$

Note that

$$
\left\|\gamma_{t}\right\|_{2}^{2}=\left\|2\left(u_{t}+1\right)^{-3} \alpha_{t}\right\|_{2}^{2} \leq O(1)\left(u_{t}+1\right)^{-6} u_{t}^{3} \leq c
$$

and

$$
\eta_{t} \leq 2\left(u_{t}+1\right)^{-3} O\left(u_{t}^{3}\right) \sqrt{\Phi} \leq c \sqrt{\Phi}
$$

for some universal constant $c$.

Let $Y_{t}$ be the process $d Y_{t}=\gamma_{t}^{T} d W_{t}$. By Theorem 6.3, there exists a Wiener process $\widetilde{W}_{t}$ such that $Y_{t}$ has the same distribution as $\widetilde{W}_{[Y]_{t}}$. Using the reflection principle for 1-dimensional Brownian motion, we have that

$$
\begin{aligned}
\mathbb{P}\left(\max _{t \in[0, T]} Y_{t} \geq \gamma\right) & \leq \mathbb{P}\left(\max _{t \in[0, c T]} \widetilde{W}_{t} \geq \gamma\right) \\
& =2 \mathbb{P}\left(\widetilde{W}_{c T} \geq \gamma\right) \leq 2 \exp \left(-\frac{\gamma^{2}}{2 c T}\right) .
\end{aligned}
$$

Therefore, we have that

$$
\mathbb{P}\left(\max _{t \in[0, T]} \Psi_{t}-\Psi_{0} \geq c \sqrt{\Phi} T+\gamma\right) \leq 2 \exp \left(-\frac{\gamma^{2}}{2 c T}\right) .
$$

Set $\Phi=4 n$. At $t=0$, we have $\operatorname{Tr}\left(u_{0} I-I\right)^{-2}=\frac{n}{4}$. Therefore, $u_{0}=\frac{3}{2}$ and $\Psi_{0}=-\frac{4}{25}$. Using the assumptions that $T \leq \frac{1}{25 c \sqrt{\Phi}}$, we have that

$$
\mathbb{P}\left(\max _{t \in[0, T]}\left(-\left(u_{t}+1\right)^{-2}\right) \geq-\frac{3}{25}+\gamma\right) \leq 2 \exp \left(-\frac{\gamma^{2}}{2 c T}\right) .
$$

The result follows from setting $\gamma=\frac{1}{120}$.

\subsection{Bounding the Size of any Initial Set}

Fix any set $E \subset \mathbb{R}^{n}$ and define $g_{t}=p_{t}(E)$.

Leмma 3.6. The random variable $g_{t}$ is a martingale satisfying

$$
d\left[g_{t}\right]_{t} \leq D^{2} g_{t}^{2} d t
$$

and

$$
d\left[g_{t}\right]_{t} \leq 30\left\|A_{t}\right\|_{\mathrm{op}} \cdot g_{t}^{2} \log ^{2}\left(\frac{1}{g_{t}}\right) d t .
$$

Using this, we can bound how fast $\log \frac{1}{g_{t}}$ changes.

LEMMA 3.7. For any $T \geq 0$ and $\gamma \geq 0$, we have that

$$
\begin{aligned}
& \mathbb{P}\left(\forall 0 \leq t \leq T: \log \frac{1}{g_{0}}+\frac{1}{2} D^{2} t+\gamma \geq \log \frac{1}{g_{t}} \geq \log \frac{1}{g_{0}}-\gamma\right) \\
& \geq 1-4 \exp \left(-\frac{\gamma^{2}}{2 T D^{2}}\right) .
\end{aligned}
$$

Now, we bound $\mathbb{E} g_{t} \sqrt{\log \frac{1}{g_{t}}}$. This is the main result of this section. 
Lemma 3.8. Assume that $n \geq 10$. There is some universal constant $c \geq 0$ such that for any measurable subset $E$ such that $p_{0}(E) \leq c$ and any $T$ such that

$$
0 \leq T \leq c \cdot \max \left(\frac{1}{D^{2}} \log \frac{1}{p_{0}(E)}, \frac{1}{\log \frac{1}{p_{0}(E)}+D}\right),
$$

we have that

$$
\mathbb{E}\left(p_{T}(E) \sqrt{\log \frac{1}{p_{T}(E)}} 1_{p_{T}(E) \leq \frac{1}{2}}\right) \geq \frac{1}{5} p_{0}(E) \sqrt{\log \frac{1}{p_{0}(E)}} .
$$

\subsection{Gaussian Case}

The next theorem can be found in [15, Thm 1.1]. We give another proof for completeness in the full version.

Theorem 3.9. Let $h(x)=f(x) e^{-\frac{t}{2}\|x\|^{2}} / \int f(y) e^{-\frac{t}{2}\|y\|^{2}} d y$ where $f: \mathbb{R}^{n} \rightarrow \mathbb{R}_{+}$is an integrable logconcave function and $t \geq 0$. Let $p(S)=\int_{S} h(x) d x$. For any $p(S) \leq \frac{1}{2}$, we have

$$
p(\partial S)=\Omega(\sqrt{t}) \cdot p(S) \sqrt{\log \frac{1}{p(S)}} .
$$

\subsection{Proof of Main Theorem}

We can now prove a bound on the isoperimetric constant.

Proof of Theorem. 3.1. By Lemma 2.2, $p_{t}$ is a martingale and therefore

$$
p(\partial E)=p_{0}(\partial E)=\mathbb{E} p_{T}(\partial E) .
$$

Next, by the definition of $p_{T}$ (1), we have that $p_{T}(x) \propto e^{c_{T}^{T} x-\frac{T}{2}\|x\|^{2}} p(x)$ and Theorem 3.9 shows that if $p_{t}(E) \leq \frac{1}{2}$, we have that

$$
p_{T}(\partial E) \gtrsim \sqrt{T} \cdot p_{t}(E) \sqrt{\log \frac{1}{p_{t}(E)}} .
$$

Hence, we have that

$$
p(\partial E) \gtrsim \sqrt{T} \cdot \mathbb{E}\left(p_{t}(E) \sqrt{\log \frac{1}{p_{t}(E)}} \cdot 1_{p_{t}(E) \leq \frac{1}{2}}\right) .
$$

Lemma 3.8 shows that if

$$
T \leq c \cdot \max \left(\frac{1}{D^{2}} \log \frac{1}{p_{0}(E)}, \frac{1}{\log \frac{1}{p_{0}(E)}+D}\right)
$$

and $p_{0}(E) \leq c$ for some small enough constant $c$, we have

$$
\begin{aligned}
p(\partial E) & \gtrsim \sqrt{T} \cdot p_{0}(E) \sqrt{\log \frac{1}{p_{0}(E)}} \\
& \gtrsim\left(\frac{\sqrt{\log \frac{1}{p_{0}(E)}}}{D}+\frac{1}{\sqrt{\log \frac{1}{p_{0}(E)}+D}}\right) p_{0}(E) \sqrt{\log \frac{1}{p_{0}(E)}} \\
& \gtrsim\left(\frac{\log \frac{1}{p_{0}(E)}}{D}+\sqrt{\frac{\log \frac{1}{p_{0}(E)}}{\log \frac{1}{p_{0}(E)}+D}}\right) p_{0}(E) \\
& \gtrsim\left(\frac{\log \frac{1}{p_{0}(E)}}{D}+\sqrt{\frac{\log \frac{1}{p_{0}(E)}}{D}}\right) p_{0}(E) .
\end{aligned}
$$

If $p_{0}(E) \geq c$, the bound simply follows from Theorem 1.2.

\section{CONSEQUENCES OF THE IMPROVED ISOPERIMETRIC INEQUALITY}

In this section, we give some consequences of the improved isoperimetric inequality (Theorem 3.1). First we note the following Cheegertype logarithmic isoperimetric inequality.

THeOREM 4.1 ([14]). Let $\mu$ be any absolutely continuous measure on $\mathbb{R}^{n}$ such that for any open subsets $A$ of $\mathbb{R}^{n}$ with $\mu(A) \leq \frac{1}{2}$,

$$
\mu(\partial A) \geq \phi \cdot \mu(A) \sqrt{\log \frac{1}{\mu(A)}} .
$$

Then, for any $f$ such that $\int_{\mathbb{R}^{n}} f^{2} d \mu=1$, we have that

$$
\int_{\mathbb{R}^{n}}|\nabla f|^{2} d \mu \gtrsim \phi^{2} \cdot \int_{\mathbb{R}^{n}} f^{2} \log f^{2} d \mu .
$$

Applying this and Theorem 3.1, we have the following result.

THEOREM 4.2. Given an isotropic logconcave distribution $p$ with diameter $D$. For any $f$ such that $\int_{\mathbb{R}^{n}} f^{2} d \mu=1$, we have that

$$
\int_{\mathbb{R}^{n}}|\nabla f(x)|^{2} p(x) d x \gtrsim \frac{1}{D} \cdot \int_{\mathbb{R}^{n}} f^{2} \log f^{2} d \mu .
$$

Now we prove Theorem 1.9, an improved concentration inequality for general Lipschitz functions and general isotropic logconcave densities.

Proof of Theorem 1.9. We first prove the statement for the function $g(x)=\|x\|$. Define

$$
E_{t}=\left\{x \text { such that }\|x\| \geq \operatorname{med}_{x \sim p}\|x\|+t\right\}
$$

and $\alpha_{t} \stackrel{\text { def }}{=} \log \frac{1}{p\left(E_{t}\right)}$. By the definition of median, we have that $\alpha_{0}=\log 2$. We first give a weak estimate on how fast $\alpha_{t}$ increases. Note that

$$
\frac{d \alpha_{t}}{d t}=-\frac{1}{p\left(E_{t}\right)} \frac{d p\left(E_{t}\right)}{d t}=\frac{p\left(\partial E_{t}\right)}{p\left(E_{t}\right)} \geq \frac{c_{1}}{n^{1 / 4}}
$$

for some universal constant $c_{1}>0$ where we used that definition of $p\left(\partial E_{t}\right)$ to get $\frac{d p\left(E_{t}\right)}{d t}=-p\left(\partial E_{t}\right)$ and Theorem 1.2 at the end. Therefore, we have that

$$
\alpha_{t+s} \geq \alpha_{t}+c_{1} \cdot s n^{-1 / 4}
$$

for all $t, s \geq 0$. To improve on this bound, we consider the distribution $p_{t}$ defined by truncating the distribution $p$ outside the set $\|x\| \geq \operatorname{med}_{x \sim p}\|x\|+t+c_{2} \sqrt{n}$ for some large enough constant $c_{2}$. By the estimate (4), we see that $p\left(E_{t}\right)$ only decreases by a tiny factor after truncation and hence

$$
\frac{p\left(\partial E_{t}\right)}{p\left(E_{t}\right)} \geq \frac{1}{2} \frac{p_{t}\left(\partial E_{t}\right)}{p_{t}\left(E_{t}\right)} .
$$

Next, we note that $p_{t}$ is almost isotropic, namely its covariance matrix $A_{t}$ satisfies $\frac{1}{2} I \leq A_{t} \leq 2 I$ (in fact, it is exponentially close to $I$ ). Although Theorem 3.1 only applies to the isotropic case, but we can always renormalize the distribution $p_{t}$ to isotropic and then normalize it back. Since the distribution $p_{t}$ is almost isotropic, such re-normalization would not change $p_{t}(\partial E)$ by more than a 
multiplicative constant. Therefore, we can apply Theorem 3.1 and get that

$$
\frac{d \alpha_{t}}{d t}=\frac{p\left(\partial E_{t}\right)}{p\left(E_{t}\right)} \geq \frac{1}{2} \frac{p_{t}\left(\partial E_{t}\right)}{p_{t}\left(E_{t}\right)} \gtrsim \sqrt{\frac{\log \frac{1}{p_{t}\left(E_{t}\right)}}{m+t}} \geq c_{3} \cdot \sqrt{\frac{\alpha_{t}}{m+t}}
$$

for some universal constant $c_{3}>0$ where $m=\operatorname{med}_{x \sim p}\|x\|+c_{2} \sqrt{n}$. Note that

$$
\frac{d \sqrt{\alpha_{t}}}{d t} \geq \frac{c_{3}}{2} \cdot \sqrt{\frac{1}{m+t}}
$$

and hence

$$
\sqrt{\alpha_{t}}-\sqrt{\alpha_{0}} \geq \frac{c_{3}}{2} \int_{0}^{t} \sqrt{\frac{1}{m+s}} d s=c_{3} \cdot(\sqrt{m+t}-\sqrt{m}) .
$$

For $t \leq m$, we have that

$$
\sqrt{\alpha_{t}}-\sqrt{\alpha_{0}} \geq c_{3} \cdot\left(\sqrt{m}+\sqrt{m} \frac{t}{3 m}-\sqrt{m}\right) \geq \frac{c_{3} \cdot t}{3 \sqrt{m}} .
$$

and hence $\alpha_{t} \geq \frac{c_{3}^{2}}{9 m} t^{2}$.

For $t \geq m$, we note that $\sqrt{1+x}-1 \geq \frac{1}{3} \sqrt{x}$ for all $x \geq 1$. Therefore,

$$
\sqrt{m+t}-\sqrt{m}=\sqrt{m}\left(\sqrt{1+\frac{t}{m}}-1\right) \geq \frac{1}{3} \sqrt{m} \sqrt{\frac{t}{m}}=\frac{1}{3} \sqrt{t} .
$$

Hence, (5) shows that $\sqrt{\alpha_{t}}-\sqrt{\alpha_{0}} \geq \frac{c_{3}}{3} \sqrt{t}$.

Combining both cases, we have that $\alpha_{t} \gtrsim \min \left(\frac{t^{2}}{\sqrt{n}}, t\right) \gtrsim \frac{t^{2}}{t+\sqrt{n}}$. Hence,

$$
\mathbb{P}_{x \sim p}\left(\|x\|-\operatorname{med}_{x \sim p}\|x\| \geq t\right) \leq \exp \left(-\Omega(1) \cdot \frac{t^{2}}{t+\sqrt{n}}\right) .
$$

By the same proof,

$$
\mathbb{P}_{x \sim p}\left(\|x\|-\operatorname{med}_{x \sim p}\|x\| \leq-t\right) \leq \exp \left(-\Omega(1) \cdot \frac{t^{2}}{t+\sqrt{n}}\right) .
$$

For a general 1-Lipschitz function, we define

$$
E_{t}=\left\{x \text { such that } g(x) \geq m_{g}+t\right\}
$$

where $m_{g}=\operatorname{med}_{x \sim p} g(x)$. Again, we define $\alpha_{t} \stackrel{\text { def }}{=} \log \frac{1}{p\left(E_{t}\right)}$ and we have $\alpha_{0}=\log 2$. To compute $\frac{p\left(\partial E_{t}\right)}{p\left(E_{t}\right)}$, we let $\zeta \geq 0$ to be chosen later and $B_{t, \zeta}$ be the ball centered at 0 with radius $c_{4} \cdot\left(\sqrt{n}+\alpha_{t}\right)+\zeta$ where $c_{4}$ is a constant. Let $p_{t, \zeta}$ be the distribution defined by $p_{t, \zeta}(A)=$ $p\left(A \cap B_{t, \zeta}\right) / p\left(B_{t, \zeta}\right)$. Choosing a large constant $c_{4}$ and using (6), we have that $p\left(B_{t, \zeta}^{c}\right) \leq \frac{p\left(E_{t}\right)}{100 n}$ and that $p_{t, \zeta}$ is almost isotropic. Since $p\left(B_{t, \zeta}^{c}\right)$ is so small, we have that $p_{t, \zeta}\left(E_{t}\right) \geq \frac{1}{2} p\left(E_{t}\right)$ and that

$$
p_{t, \zeta}\left(\partial E_{t}\right) \leq \frac{p\left(\partial\left(E_{t} \cap B_{t, \zeta}\right)\right)}{p\left(B_{t, \zeta}\right)} \leq 2 p\left(\partial E_{t}\right)+2 p\left(\partial B_{t, \zeta}\right) .
$$

Hence,

$$
\frac{p\left(\partial E_{t}\right)}{p\left(E_{t}\right)} \geq \frac{1}{4} \frac{p_{t, \zeta}\left(\partial E_{t}\right)}{p_{t, \zeta}\left(E_{t}\right)}-\frac{p_{t, \zeta}\left(\partial B_{t, \zeta}\right)}{p\left(E_{t}\right)} .
$$

Since $p_{t, \zeta}$ is almost isotropic, Theorem 3.1 gives that

$$
\frac{p_{t, \zeta}\left(\partial E_{t}\right)}{p_{t, \zeta}\left(E_{t}\right)} \gtrsim \sqrt{\frac{\log \frac{1}{p_{t, \zeta}\left(E_{t}\right)}}{\sqrt{n}+\alpha_{t}+\zeta}} \geq c_{5} \sqrt{\frac{\alpha_{t}}{\sqrt{n}+\alpha_{t}+\zeta}}
$$

for some universal constant $0<c_{5}<1$. To bound the second term $p_{t, \zeta}\left(\partial B_{t, \zeta}\right)$, we note that

$$
\begin{aligned}
\int_{0}^{1 / c_{5}^{2}} p_{t, \zeta}\left(\partial B_{t, \zeta}\right) d \zeta & \leq 2 \int_{0}^{1 / c_{5}^{2}} p\left(\partial B_{t, \zeta}\right) d \zeta \leq 2 p\left(B_{t, 0}^{c}\right) \\
& \leq \frac{p\left(E_{t}\right)}{50 n} .
\end{aligned}
$$

Hence, there is $\zeta$ between 0 and $1 / c_{5}^{2}$ such that $p_{t, \zeta}\left(\partial B_{t, \zeta}\right) \leq$ $\frac{p\left(E_{t}\right)}{50 n} c_{5}^{2}$.

Using this, (7) and (8), we have that

$$
\begin{aligned}
\frac{p\left(\partial E_{t}\right)}{p\left(E_{t}\right)} & \geq \frac{c_{5}}{4} \sqrt{\frac{\alpha_{t}}{\sqrt{n}+\alpha_{t}+1 / c_{5}^{2}}}-\frac{c_{5}^{2}}{50 n} \\
& \geq \frac{c_{5}}{8} \sqrt{\frac{\alpha_{t}}{\sqrt{n}+\alpha_{t}+1 / c_{5}^{2}}}+\frac{c_{5}}{16} \sqrt{\frac{1}{\sqrt{n}+1 / c_{5}^{2}}}-\frac{c_{5}^{2}}{50 n} \\
& \geq \frac{c_{5}}{8} \sqrt{\frac{\alpha_{t}}{\sqrt{n}+\alpha_{t}+1 / c_{5}^{2}}} .
\end{aligned}
$$

Next, we relate $\frac{p\left(\partial E_{t}\right)}{p\left(E_{t}\right)}$ with $d p\left(E_{t}\right)$. Note that for any $x$ such that $\|x-y\|_{2} \leq h$ and $y \in E_{t}$, we have that

$$
g(x) \geq m_{g}+t-h
$$

because $g$ is 1-Lipschitz. Therefore, we have $B\left(E_{t}, h\right) \subset E_{t-h}$. Therefore,

$$
\begin{aligned}
-\frac{d p\left(E_{t}\right)}{d t} & =\lim _{h \rightarrow 0} \frac{p\left(\left\{g(x) \geq \operatorname{med}_{x \sim p} g(x)+t-h\right\}\right)-p\left(E_{t}\right)}{h} \\
& \geq \lim _{h \rightarrow 0} \frac{p\left(B\left(E_{t}, h\right)\right)-p\left(E_{t}\right)}{h}=p\left(\partial E_{t}\right) .
\end{aligned}
$$

Using this with (9), we have that

$$
\frac{d \alpha_{t}}{d t}=-\frac{1}{p\left(E_{t}\right)} \frac{d p\left(E_{t}\right)}{d t} \geq \frac{p\left(\partial E_{t}\right)}{p\left(E_{t}\right)} \geq \frac{c_{5}}{8} \sqrt{\frac{\alpha_{t}}{\sqrt{n}+\alpha_{t}+1 / c_{5}^{2}}} .
$$

Solving this equation, we again have that $\alpha_{t} \gtrsim \frac{t^{2}}{t+\sqrt{n}}$. This proves that

$$
\mathbb{P}_{x \sim p}\left(g(x)-\operatorname{med}_{x \sim p} g(x) \geq t\right) \leq \exp \left(-\Omega(1) \cdot \frac{t^{2}}{t+\sqrt{n}}\right) .
$$

The case of $g(x)-\operatorname{med}_{x \sim p} g(x) \leq-t$ is the same by taking the negative of $g$. This proves the theorem. To replace $\operatorname{med}_{x \sim p} g(x)$ by $\mathbb{E}_{x \sim p} g(x)$, we simply need to use the concentration we just proved to show that $\left|\mathbb{E}_{x \sim p} g(x)-\operatorname{med}_{x \sim p} g(x)\right| \lesssim n^{\frac{1}{4}}$.

\section{OPTIMALITY OF THE BOUNDS}

Lemma 5.1. For any $\frac{n}{2} \geq D \geq 2 \sqrt{n}$, there exists an isotropic logconcave distribution with diameter $D$ such that its log-Cheeger constant is $O(1 / \sqrt{D})$ and its log-Sobolev constant is $O(1 / D)$.

In fact, we get a nearly lower tight bound on the mixing time of the ball walk, from an arbitrary start, in terms of the number of proper steps. Recall that by a proper step we mean steps where the current point changes, not counting the steps that are discarded due to the rejection probability. In our lower bound example, the local conductance, i.e., the probability of a proper step, is at least a 


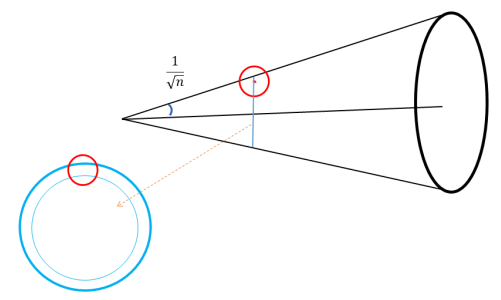

Figure 1: The lower bound construction

constant everywhere and so the total number of steps in expectation is within a constant factor of the number of proper steps.

Lemma 5.2. For any $\frac{n}{2} \geq D \geq 2 \sqrt{n}$, there exists an isotropic convex body with diameter $D$ such that ball walk mixes in $\widetilde{\Omega}\left(n^{2} D\right)$ proper steps.

Both theorems are based on the following cone

$$
K=\left\{x: 0 \leq x_{1} \leq n, \sum_{i=2}^{n} x_{i}^{2} \leq \frac{1}{n} x_{1}^{2}\right\},
$$

and the truncated cone

$$
K_{D}=K \cap\left\{x:\left(x_{1}-n\right)^{2}+\sum_{i=2}^{n} x_{i}^{2} \leq D^{2}\right\} .
$$

Proof of Lemma 5.1. The convex body $K_{D}$ is nearly isotropic and has diameter $D$. Let

$$
t_{0}=n-\sqrt{D^{2}-n} .
$$

Consider the subset $S=K \cap\left\{t_{0} \leq x_{1} \leq t_{0}+1\right\}$. Note that $S$ is fully contained in $K_{D}$ and that

$$
\begin{aligned}
p & =\frac{\operatorname{vol}(S)}{\operatorname{vol}\left(K_{D}\right)} \approx \frac{\operatorname{vol}(S)}{\operatorname{vol}(K)} \\
& =\left(\frac{t_{0}+1}{n}\right)^{n-1}-\left(\frac{t_{0}}{n}\right)^{n-1} \\
& \approx e^{-D} .
\end{aligned}
$$

On the other hand, the expansion of $S$ is at most 2. Therefore, the log-Cheeger constant $\kappa$ of $K_{D}$ must satisfy

$$
\kappa \sqrt{\log \frac{1}{p}} \leq 2
$$

or $\kappa=O\left(D^{-\frac{1}{2}}\right)$ as claimed. It is known that the log-Sobolev constant $\rho=\Theta\left(\kappa^{2}\right)$ (see e.g., [14]). This gives the second claim.

\section{PRELIMINARIES}

In this section, we review some basic definitions and theorems that we use in the proofs.

\subsection{Stochastic Calculus}

Given real-valued stochastic processes $x_{t}$ and $y_{t}$, the quadratic variations $[x]_{t}$ and $[x, y]_{t}$ are real-valued stochastic processes defined by

$$
[x]_{t}=\lim _{|P| \rightarrow 0} \sum_{n=1}^{\infty}\left(x_{\tau_{n}}-x_{\tau_{n-1}}\right)^{2}
$$

and

$$
[x, y]_{t}=\lim _{|P| \rightarrow 0} \sum_{n=1}^{\infty}\left(x_{\tau_{n}}-x_{\tau_{n-1}}\right)\left(y_{\tau_{n}}-y_{\tau_{n-1}}\right),
$$

where $P=\left\{0=\tau_{0} \leq \tau_{1} \leq \tau_{2} \leq \cdots \uparrow t\right\}$ is a stochastic partition of the non-negative real numbers, $|P|=\max _{n}\left(\tau_{n}-\tau_{n-1}\right)$ is called the mesh of $P$ and the limit is defined using convergence in probability. Note that $[x]_{t}$ is non-decreasing with $t$ and $[x, y]_{t}$ can be defined as

$$
[x, y]_{t}=\frac{1}{4}\left([x+y]_{t}-[x-y]_{t}\right) .
$$

If the processes $x_{t}$ and $y_{t}$ satisfy the SDEs

$$
d x_{t}=\mu\left(x_{t}\right) d t+\sigma\left(x_{t}\right) d W_{t}
$$

and $d y_{t}=v\left(y_{t}\right) d t+\eta\left(y_{t}\right) d W_{t}$ where $W_{t}$ is a Wiener process, we have that

$$
[x]_{t}=\int_{0}^{t} \sigma^{2}\left(x_{s}\right) d s, \quad[x, y]_{t}=\int_{0}^{t} \sigma\left(x_{s}\right) \eta\left(y_{s}\right) d s
$$

and

$$
d[x, y]_{t}=\sigma\left(x_{t}\right) \eta\left(y_{t}\right) d t .
$$

For a vector-valued SDE $d x_{t}=\mu\left(x_{t}\right) d t+\Sigma\left(x_{t}\right) d W_{t}$ and $d y_{t}=$ $v\left(y_{t}\right) d t+M\left(y_{t}\right) d W_{t}$, we have that

$$
\left[x^{i}, x^{j}\right]_{t}=\int_{0}^{t}\left(\Sigma\left(x_{s}\right) \Sigma^{T}\left(x_{s}\right)\right)_{i j} d s
$$

and

$$
d\left[x^{i}, y^{j}\right]_{t}=\left(\Sigma\left(x_{t}\right) M^{T}\left(y_{t}\right)\right)_{i j} d t .
$$

LEMMA 6.1 (ITO's FORMULA). Let $x$ be a semimartingale and $f$ be a twice continuously differentiable function, then

$$
d f\left(x_{t}\right)=\sum_{i} \frac{d f\left(x_{t}\right)}{d x^{i}} d x^{i}+\frac{1}{2} \sum_{i, j} \frac{d^{2} f\left(x_{t}\right)}{d x^{i} d x^{j}} d\left[x^{i}, x^{j}\right]_{t} .
$$

The next two lemmas are well-known facts about Wiener processes; first the reflection principle.

LEMMA 6.2 (REFLECTION PRINCIPLE). Given a Wiener process $W(t)$ and $a, t \geq 0$, then we have that

$$
\mathbb{P}\left(\sup _{0 \leq s \leq t} W(s) \geq a\right)=2 \mathbb{P}(W(t) \geq a)
$$

Second, a decomposition lemma for continuous martingales.

Theorem 6.3 (Dambis, Dubins-Schwarz theorem). Every continuous local martingale $M_{t}$ is of the form

$$
M_{t}=M_{0}+W_{[M]_{t}} \text { for all } t \geq 0
$$

where $W_{s}$ is a Wiener process. 


\subsection{Logconcave Functions and Isoperimetry}

We say a logconcave distribution is nearly isotropic if its covariance matrix $A$ satisfies $\Omega(1) \cdot I \leq A \leq O(1) \cdot I$. The following lemma about logconcave densities is folklore, see e.g., [19].

LEMma 6.4 (LogConcAVE MOMENTs). Given a logconcave density $p$ in $\mathbb{R}^{n}$, and any integer $k \geq 1$,

$$
\mathbb{E}_{x \sim p}\|x\|^{k} \leq(2 k)^{k}\left(\mathbb{E}_{x \sim p}\|x\|^{2}\right)^{k / 2} .
$$

Theorem 6.5 (Poincaré constant $[5,22]$ ). For any logconcave density $p$ in $\mathbb{R}^{n}$ and any function $g$ in $\mathbb{R}^{n}$, we have

$$
\operatorname{Var}_{p}(g(x)) \lesssim \psi_{p}^{-2} \cdot \mathbb{E}_{p}\left(\|\nabla g(x)\|_{2}^{2}\right)
$$

\section{ACKNOWLEDGEMENT}

This work was supported in part by NSF Awards CCF-1563838, CCF-1717349, CCF-1740551 and CCF-1749609. We thank Ravi Kannan and Ronen Eldan for inspiring discussions, and Ronen for his wonderful invention of stochastic localization.

\section{REFERENCES}

[1] Zeyuan Allen-Zhu, Zhenyu Liao, and Lorenzo Orecchia. 2015. Spectral sparsification and regret minimization beyond matrix multiplicative updates. In Proceedings of the forty-seventh annual ACM symposium on Theory of computing ACM, 237-245.

[2] Jean-Yves Audibert, Sébastien Bubeck, and Gábor Lugosi. 2013. Regret in online combinatorial optimization. Mathematics of Operations Research 39, 1 (2013), $31-45$.

[3] Joshua D. Batson, Daniel A. Spielman, and Nikhil Srivastava. 2012. TwiceRamanujan Sparsifiers. SIAM f. Comput. 41, 6 (2012), 1704-1721. https: //doi.org/10.1137/090772873

[4] Silouanos Brazitikos, Apostolos Giannopoulos, Petros Valettas, and BeatriceHelen Vritsiou. 2014. Geometry of isotropic convex bodies. Vol. 196. American Mathematical Society Providence.

[5] Jeff Cheeger. 1969. A lower bound for the smallest eigenvalue of the Laplacian. Princeton Univ. Press, 195-199.

[6] P. Diaconis and L. Saloff-Coste. 1996. Logarithmic Sobolev inequalities for finite Markov chains. Ann. Appl. Probab. 6, 3 (08 1996), 695-750. https://doi.org/10. 1214/aoap/1034968224
[7] R. Eldan. 2013. Thin shell implies spectral gap up to polylog via a stochastic localization scheme. Geometric and Functional Analysis 23 (2013), 532-569.

[8] Alan Frieze and Ravi Kannan. 1999. Log-Sobolev inequalities and sampling from log-concave distributions. Ann. Appl. Probab. 9, 1 (02 1999), 14-26. https: //doi.org/10.1214/aoap/1029962595

[9] M. Gromov and V. D. Milman. 1983. A topological application of the isoperimetric inequality. Amer. F. Math. 105, 4 (1983), 843-854.

[10] Olivier Guedon and Emanuel Milman. 2011. Interpolating Thin-Shell and Sharp Large-Deviation Estimates for Isotropic Log-Concave Measures. Geometric and Functional Analysis 21, 5 (2011), 1043-1068. https://doi.org/10.1007/ s00039-011-0136-5

[11] Ravi Kannan, László Lovász, and Ravi Montenegro. 2006. Blocking Conductance and Mixing in Random Walks. Combinatorics, Probability \& Computing 15, 4 (2006), 541-570. https://doi.org/10.1017/S0963548306007504

[12] R. Kannan, L. Lovász, and M. Simonovits. 1995. Isoperimetric Problems for Convex Bodies and a Localization Lemma. Discrete \& Computational Geometry 13 (1995), 541-559.

[13] R. Kannan, L. Lovász, and M. Simonovits. 1997. Random walks and an $O^{*}\left(n^{5}\right)$ volume algorithm for convex bodies. Random Structures and Algorithms 11 (1997), $1-50$.

[14] Michel Ledoux. 1994. A simple analytic proof of an inequality by P. Buser. Proceedings of the American mathematical society 121, 3 (1994), 951-959.

[15] Michel Ledoux. 1999. Concentration of measure and logarithmic Sobolev inequalities. SÃ®minaire de probabilit $\tilde{A} \odot s$ de Strasbourg 33 (1999), 120-216. http://eudml.org/doc/114006

[16] Yin Tat Lee and He Sun. 2015. Constructing linear-sized spectral sparsification in almost-linear time. In Foundations of Computer Science (FOCS), 2015 IEEE 56th Annual Symposium on. IEEE, 250-269.

[17] Yin Tat Lee and He Sun. 2017. An SDP-based algorithm for linear-sized spectral sparsification. In Proceedings of the 49th Annual ACM SIGACT Symposium on Theory of Computing. ACM, 678-687.

[18] Yin Tat Lee and Santosh Srinivas Vempala. 2017. Eldan's Stochastic Localization and the KLS Hyperplane Conjecture: An Improved Lower Bound for Expansion. In Proc. of IEEE FOCS

[19] L. Lovász and S. Vempala. 2007. The geometry of logconcave functions and sampling algorithms. Random Structures and Algorithms 30, 3 (2007), 307-358.

[20] G. Paouris. 2006. Concentration of mass on convex bodies. Geometric and Functional Analysis 16 (2006), 1021-1049.

[21] Nikhil Srivastava, Roman Vershynin, et al. 2013. Covariance estimation for distributions with 2+eps moments. The Annals of Probability 41, 5 (2013), 30813111.

[22] V.G.Maz'ja. 1960. Classes of domains and imbedding theorems for function spaces. Dokl. Acad. Nauk SSSR (Engl. transl. Soviet Math. Dokl., 1 (1961) 882-885) 3 (1960), 527-530. 